\title{
Anti-endotoxin Properties of Polymyxin B-immobilized Fibers
}

\author{
Tohru Tani, Tomoharu Shimizu, Masaji Tani, \\ Hisataka Shoji, and Yoshihiro Endo
}

\begin{abstract}
Polymyxin B is an antibiotic that shows strong bactericidal activity against Gram-negative bacteria, by binding to and inactivating endotoxin. Systemic administration of polymyxin $\mathrm{B}$ in humans is restricted because of its nephrotoxicity and neurotoxicity, and this compound was therefore considered a strong candidate ligand for the extracorporeal selective adsorption of circulating endotoxin in the blood. Toraymyxin ${ }^{\circledR}$ is a direct hemoperfusion column that uses polymyxin B attached to an insoluble carrier to bind endotoxin in the blood. In 1994, the Japanese National Health Insurance system approved the use of Toraymyxin for the treatment of endotoxemia and septic shock.
\end{abstract}

Authors Tohru Tani and Tomoharu Shimizu have equally contributed to this chapter.

T. Tani $(\bowtie)$

Biomedical Innovation Center, Shiga University

of Medical Science, Otsu, Shiga, Japan

Department of Surgery, Shiga University

of Medical Science, Otsu, Shiga, Japan

e-mail: tan@belle.shiga-med.ac.jp

T. Shimizu $\cdot$ M. Tani $\cdot$ H. Shoji

Department of Surgery, Shiga University

of Medical Science, Otsu, Shiga, Japan

Y. Endo

Department of Clinical Nursing, Shiga University

of Medical Science, Otsu, Shiga, Japan
In this chapter, we will review the development, clinical use, and efficacy of Toraymyxin, examine the structure of the Toraymyxin column, and comment on the current position of Toraymyxin in the treatment of severe sepsis and septic shock. We will also highlight some potential new applications of Toraymyxin for pulmonary diseases.

\section{Keywords}

Polymyxin $\cdot$ Lipopolysaccharide ·

Endotoxemia $\cdot$ Septic shock $\cdot$ Toraymyxin

\subsection{Introduction}

Endotoxin is one of major constituents of the cell wall in Gram-negative bacteria. Endotoxin is recognized through toll-like receptor-4 as a pathogen-associated molecular component that activates macrophages and other leukocytes to produce various inflammatory mediators. Endotoxin plays a major role in the development of toxic symptoms in Gram-negative bacterial infections. A recent review showed that codetection of Gram-negative bacteria and endotoxemia is strongly predictive of increased risk of mortality compared to detection of neither [1]. It is therefore a reasonable therapeutic approach to evaluate and remove circulating endotoxin in 
the blood of patients with Gram-negative bacterial infections.

Polymyxin B is a polycationic antibiotic that binds to and inactivates endotoxin, thereby neutralizing endotoxin-associated toxicity in humans [2]. Systemic administration of polymyxin B in humans is restricted because of its nephrotoxicity and neurotoxicity, and this compound was considered a strong candidate ligand for the extracorporeal selective adsorption of circulating endotoxin in the blood.

The selective removal of endotoxin from blood has been discussed since the 1970s, when Nolan and Ali first demonstrated the adsorption of endotoxin via the ion-exchange resin cholestyramine [3]. Various non-selective adsorbents such as charcoal or ion-exchange resins have since been considered, but a new selective adsorbent was needed for the specific and efficient removal of circulating endotoxin from blood. Sepharose $4 \mathrm{~B}$ beads to which polymyxin B was covalently immobilized were shown to be effective adsorbents for the removal of endotoxin in a rat model and in human hemodialysis systems [4, 5]. Polyethylenimine [6] and albumin [7], rather than polymyxin $\mathrm{B}$, have also been used as ligands for the selective adsorption of endotoxin.

Initial research into polymyxin B-immobilized fibers began in 1981 at the Department of Surgery, Shiga University of Medical Science, Japan, as a collaboration between our research group and Toray Medical Co., Ltd. (Chiba, Japan) (Fig. 19.1). In 1982, the preliminary results of this research were published, demonstrating that polymyxin B-immobilized fibers reduce endotoxin activity in saline solution, as measured using a semi-quantitative limulus amebocyte lysate (LAL) assay.

In 1983, we began in vitro and in vivo experimental studies. A pre-clinical study showed that selective removal of endotoxin from the blood by extracorporeal direct hemoperfusion with

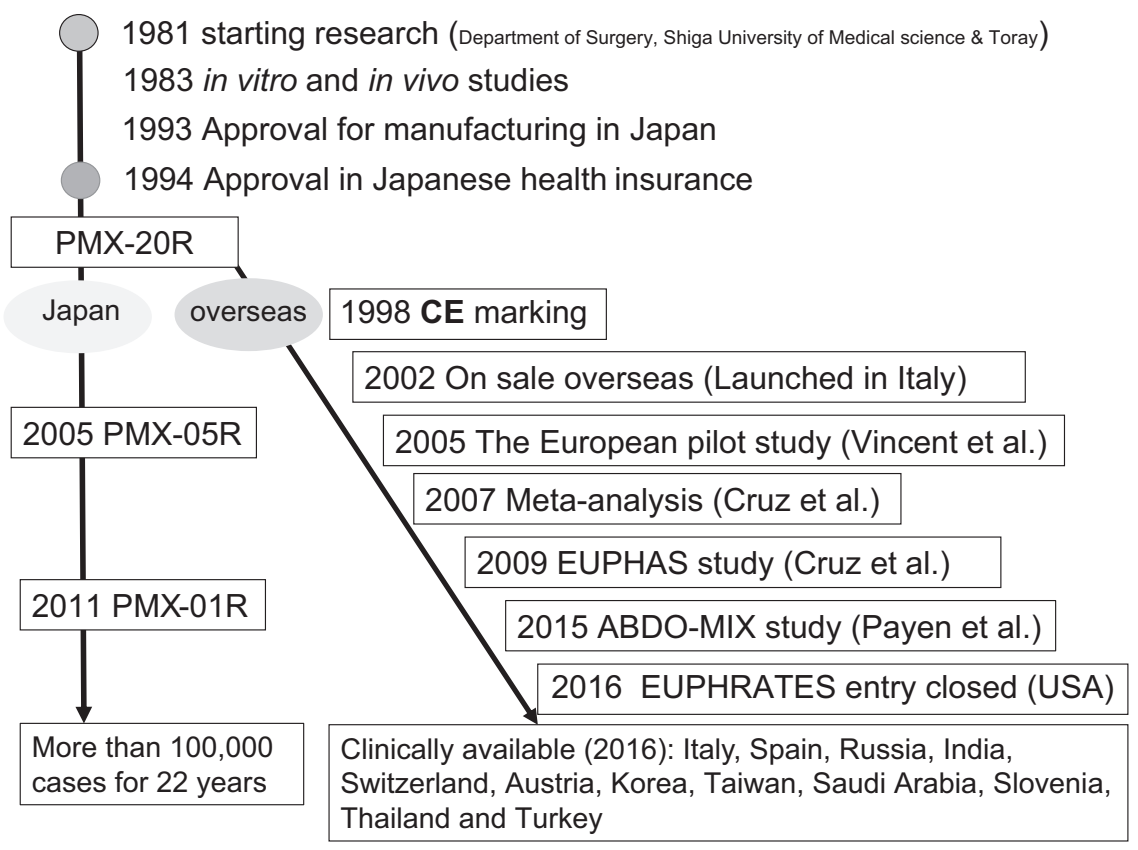

Fig. 19.1 History of the development of Toraymyxin EUPHAS: Early use of polymyxin B hemoperfusion in abdominal sepsis

ABDO-MIX trial: Effects of hemoperfusion with a polymyxin $\mathrm{B}$ membrane in peritonitis with septic shock
EUPHRATES trial: Evaluating the use of polymyxin B hemoperfusion in a randomized controlled trial of adults treated for endotoxemia and septic shock 
polymyxin B-immobilized fibers resulted in improved survival in an endotoxemia canine model [8]. Survival also improved in a Gramnegative bacterium (Escherichia coli) intravenous administration canine model after extracorporeal hemoperfusion with polymyxin B-immobilized fibers, indicating the potential for use thereof in humans [9].

The polymyxin B-immobilized fibers blood purification column, Toraymyxin ${ }^{\circledR}$, was developed as a medical device to be used in conjunction with direct hemoperfusion to remove circulating endotoxin from the blood in humans. A phase I clinical study was initiated in 1989, and in 1994 the first clinical report of 16 patients with septic multiple organ failure treated with direct hemoperfusion with Toraymyxin (PMX) was published [10]. PMX for $2 \mathrm{~h}$ significantly decreased the level of circulating endotoxin from 76 to $21 \mathrm{pg} / \mathrm{mL}$. Furthermore, the patients' hyperdynamic state (in terms of cardiac index), which is characteristic of endotoxic shock, returned to normal after treatment [10]. Regarding the technical term for Toraymyxin, we have suggested defining "PMX" as "direct hemoperfusion with the polymyxin B-immobilized fiber column (Toraymyxin)".

In 1994, Toraymyxin was adopted by the Japanese National Health Insurance system for the treatment of endotoxemia and septic shock [11]. Since then, Toraymyxin has been used safely in more than 100,000 cases in emergency and intensive care units in Japan. Three different columns are currently available for clinical use in Japan: PMX-20R for use in adults, PMX-05R for use in children, and PMX-01R for use in babies (Fig. 19.1).

In 1998, Toraymyxin received CE mark approval in Europe. In 2005, the results of the first randomized controlled trial (RCT) in Europe were published, showing that treatment with Toraymyxin is safe, and improves cardiac and renal dysfunction due to sepsis or septic shock [12]. In 2007, the findings of a meta-analysis demonstrated the favorable effects of PMX [13]. In 2009, the results of the second RCT in Europe, the EUPHAS study (Early Use of Polymyxin B Hemoperfusion in Abdominal Sepsis), which was conducted in Italy, were pub- lished, showing that Toraymyxin treatment results in a significant reduction in sepsis-associated mortality [14]. A large RCT, the ABDO-MIX trial in France, has failed to show survival benefit and improvement in organ failure with Toraymyxin treatment compared to conventional treatment of peritonitis-induced septic shock [15]. Recruitment into another large RCT, the EUPHRATES trial in the USA, is now closed and the results showed that polymyxin B hemoperfusion plus conventional medical therapy did not reduce mortality at 28 days in patients with septic shock and high endotoxin activity (Fig. 19.1, ClinicalTrials.gov Identifier: NCT01046669). Toraymyxin is currently available for use in clinical settings in Italy, Spain, Russia, India, Switzerland, Austria, Korea, Taiwan, Saudi Arabia, Slovenia, Thailand, and Turkey.

In this chapter, we will review the development, clinical use, and efficacy of Toraymyxin, examine the structure of the Toraymyxin column, and comment on the current position of Toraymyxin in the treatment of severe sepsis and septic shock. We will also highlight some potential new applications for Toraymyxin.

\subsection{Development of Polymyxin B-immobilized Fibers}

\subsubsection{Polymyxin B and Endotoxin}

Polymyxin B has strong bactericidal activity against Gram-negative bacteria. The bactericidal properties of polymyxin $\mathrm{B}$ are discussed in more detail in other chapters. Polymyxin B also neutralizes the lethal toxicity, limulus gelation activity, and hemodynamic effects of endotoxin, a major component of the outer membrane of Gramnegative bacteria, by binding to the lipid A domain, which is the active center of the endotoxin molecule [16]. The protective effects of polymyxin B against endotoxemia and septicemia have been demonstrated in various animal models [17-19].

Electron microscopy imaging has revealed that when endotoxin is exposed to polymyxin B, its usual ribbon-like structure partially or fully disaggregates [20]. The detergent-like activity of polymyxin $\mathrm{B}$ arises from its ability to prompt the 
dissociation of endotoxin's micellar structure [21]. Polymyxin B has been shown to bind to the lipopolysaccharide of Salmonella typhimurium at the negatively charged 2-keto-3deoxyoctulosonate lipid A region of the lipopolysaccharide molecule. This is achieved via electrostatic, and possibly hydrophobic, interactions at a stoichiometric ratio of one polymyxin B molecule to one lipopolysaccharide monomer unit [22]. Furthermore, Vesentini et al. demonstrated that short-range interactions between polymyxin B and endotoxin are mediated mainly via hydrophobic forces, whereas long-term complex formation is mediated via ionic forces. The interaction energy occurring in each molecular complex was calculated at different intermolecular distances, and the binding forces were estimated by fitting interaction energy data. Maximum binding forces calculated via molecular mechanics for the polymyxin B-endotoxin complex range from 1.39 to $3.79 \mathrm{nN}$ [23].

Together, these studies have clarified that polymyxin $\mathrm{B}$ binds to and detoxifies endotoxin, and that it is through this binding that polymyxin B exerts its anti-microbial activity.

\subsubsection{Polymyxin B-immobilized Fibers}

To enable selective adsorption of circulating endotoxin in the blood, polymyxin B was covalently immobilized on the surface of polystyrenederived, polypropylene-reinforced conjugated carrier fibers (Fig. 19.2). $\alpha$-Chloroacetoamide methyl groups were chemically introduced into the polystyrene molecule to provide a moiety to which the polymyxin B could be fixed [24]. The endotoxin adsorption capacity of polymyxin B-immobilized fibers has been shown to be almost the same as that of the ion-exchange resin IRA-938 (Fig. 19.3a, b) [24].

The endotoxin detoxification capacity of polymyxin B-immobilized fibers in vitro changes depending on the number of residual primary amino groups in the immobilized polymyxin B molecule

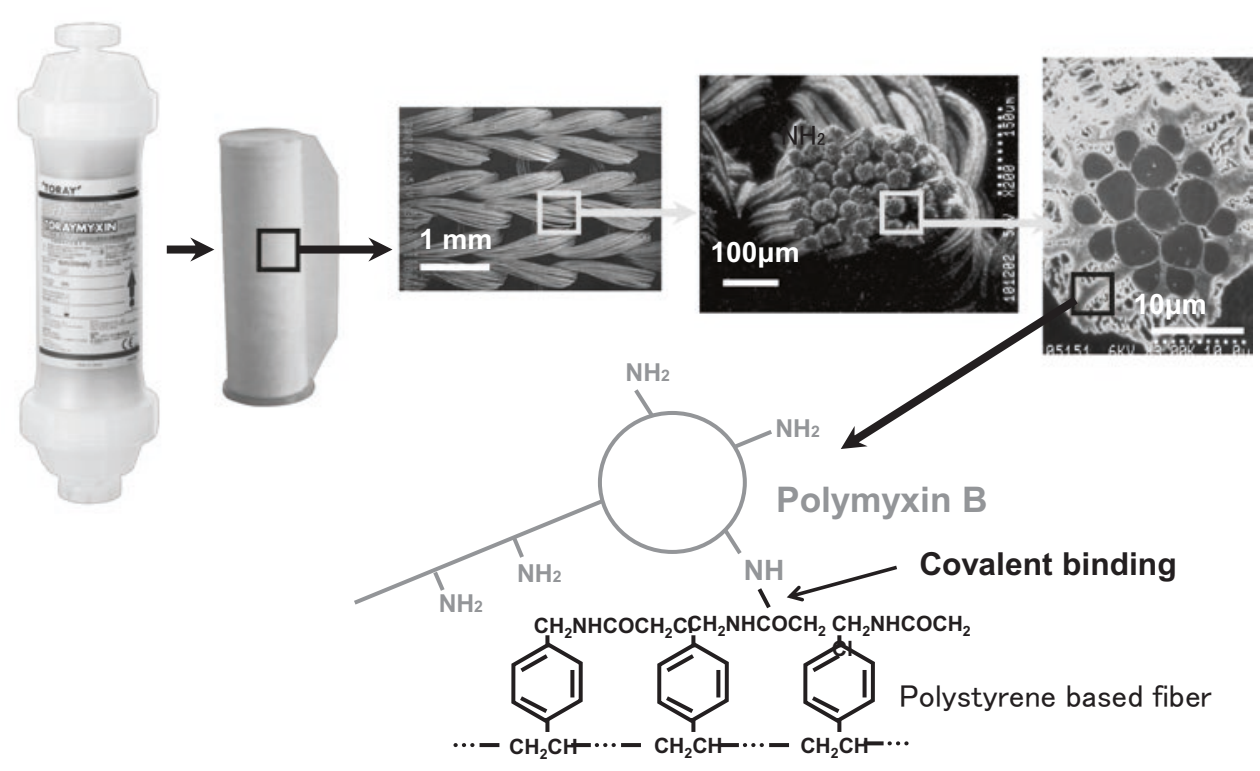

Fig. 19.2 Physical structure of a Toraymyxin cartridge and of the knitted fabric roll of polymyxin B-immobilized fibers. (Provided by Toray Medical Co., Ltd.)

The Toraymyxin cartridge contains a roll of knitted fibers. Each fiber consists of a bundle of ultra-fine fibers with a diameter of approximately $20 \mu \mathrm{m}$. The polymyxin B molecules are covalently bound onto the fiber surface and therefore do not leak into the patient

Molecular conformation is shown. Polymyxin B was covalently bound to polystyrene-based fibers 
Fig. 19.3 Endotoxin adsorption capacity of polymyxin

B-immobilized fiber or IRA-938 data from Shoji et al. [24]. Two grams of polymyxin

B-immobilized fibers (polymyxin

B-immobilized fibers, solid line) or ionexchange resin (IRA-938, dotted line) were incubated with $30 \mathrm{~mL}$ of various concentrations of endotoxin in (a) aqueous solution or (b) bovine serum solution
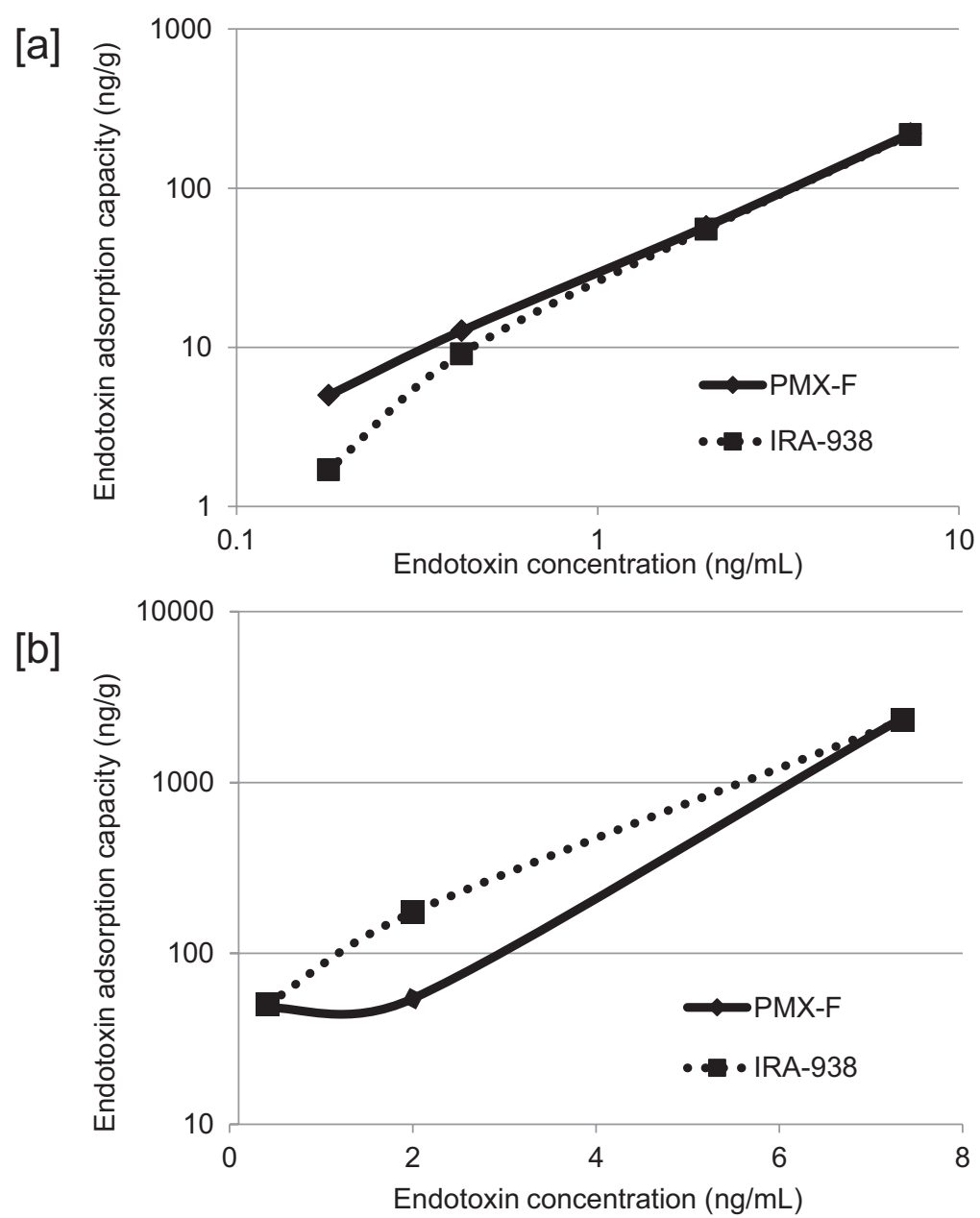

(Fig. 19.4a) [24]. In an in vivo canine experiment, the survival rate of endotoxin-challenged dogs was higher when the immobilized polymyxin B had a large number of residual primary amino groups (Fig. 19.4b) [24]. These results suggested that polymyxin B-immobilized fibers had a maximal endotoxin detoxification capacity when three primary amino groups were left unbound from the carrier fiber. Furthermore, concentrations of immobilized polymyxin B above $3.5 \mu \mathrm{g} / \mathrm{mg}$ of fiber were suggested to be optimal. Because the primary amino groups are positively charged, it is likely that they play a major role in the ionic binding of polymyxin B to endotoxin [24].

In addition to detoxifying the limulus activity of endotoxin, polymyxin B-immobilized fibers have also been shown to neutralize the pyrogenic activity of endotoxin solution in a rabbit pyrogen test. Therefore, polymyxin B-immobilized fibers are also effective in removing pyrogenic agents from serum (Fig. 19.5) [24].

\subsubsection{Endotoxin Adsorption Capacity and Bactericidal Activity of Polymyxin B-immobilized Fibers In Vitro}

When polymyxin B-immobilized fibers, or carrier fibers alone, were incubated with synthetic lipid A in vitro, polymyxin B-immobilized fibers effectively adsorbed synthetic lipid A, whereas the carrier fibers alone did not, as assessed using an LAL assay, showing that the capability of polymyxin B to bind to lipid A is retained even when polymyxin B is immobilized (Fig. 19.6a) 
[a]

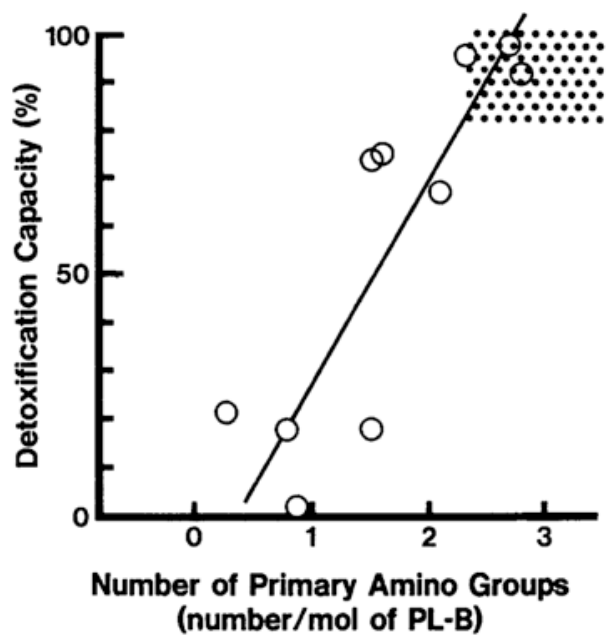

[b]

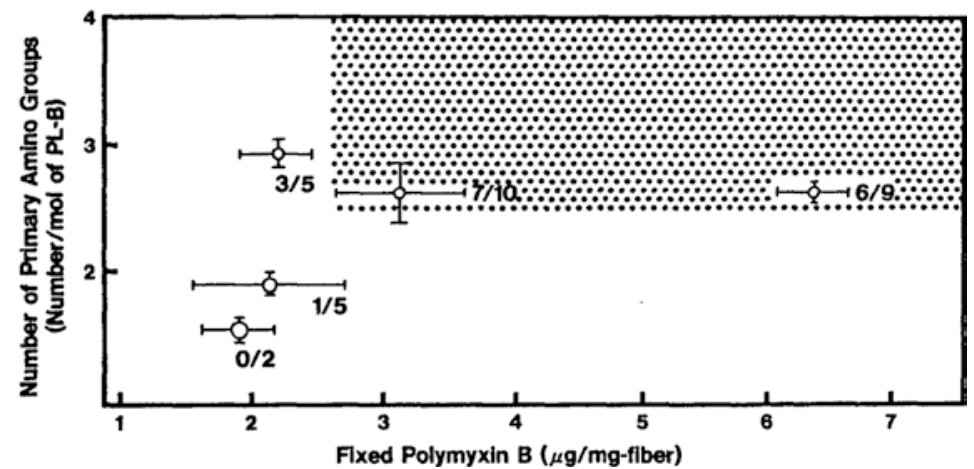

Fig. 19.4 Relationship between the residual number of primary amino groups in the immobilized polymyxin B molecule

(a) Relationship between the residual number of primary amino groups in the immobilized polymyxin B molecule and its detoxification capacity

Polymyxin B-immobilized fibers were added to a bovine serum solution containing lipopolysaccharides, and incubated with continuous stirring for $2 \mathrm{~h}$. Endotoxin concentration was measured via limulus amebocyte lysate assay. Polymyxin B-immobilized fibers had the best endotoxin

[24]. The lipid A portion of endotoxin is located in the least variable region of the lipopolysaccharide molecule, meaning that it does not change from species to species, or from strain to strain. Polymyxin B-immobilized fibers can adsorb various types of endotoxins with different O-side chains or chemotypes, indicating that immobilized polymyxin B binds specifically to the lipid A portion of the LPS molecule (Fig. 19.6b) [24]. detoxification capacity when three amino groups were left in the immobilized polymyxin B molecule bound to the carrier fiber. (Reproduced from Shoji et al. [24])

(b) Relationship between the number of residual amino groups in fixed immobilized polymyxin B and the rate of survival in endotoxin-challenged dogs

The number of residual primary amino groups in the immobilized polymyxin B had a greater overall effect on the survival of dogs than did the quantity of immobilized polymyxin B used. (Reproduced from Shoji et al. [24])

Polymyxin B-immobilized fibers also show bactericidal activity. Polymyxin B-immobilized fibers were added to a phosphate buffer solution containing Pseudomonas aeruginosa, and changes in bacterial cell count were assessed under continual stirring for $9 \mathrm{~h}$. Bacterial cell counts decreased sharply from approximately $10^{7}$ to $10^{3} \mathrm{CFU} / \mathrm{mL}$ (Fig. 19.6c) [24].

Together, these results clearly demonstrate that immobilized polymyxin B retains the endo- 


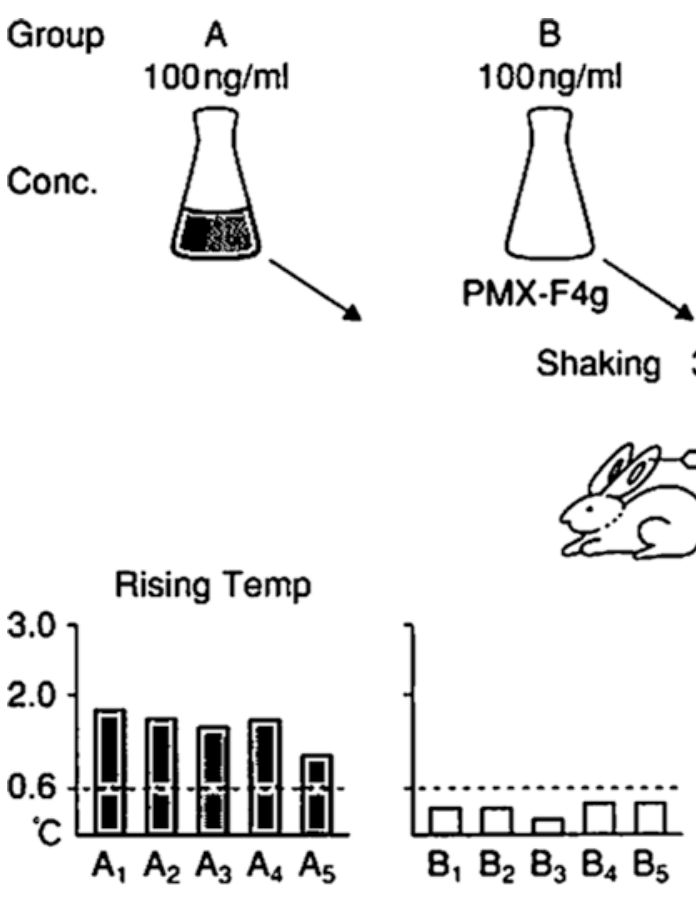

Fig. 19.5 Endotoxin removal by polymyxin B-immobilized fibers examined in a rabbit pyrogen test Polymyxin B-immobilized fibers (PMX-F) were incubated with various concentrations of endotoxin in an

toxin binding and bactericidal properties of free polymyxin B [24].

\subsubsection{Endotoxin Adsorption Capacity of Polymyxin B-immobilized Fibers In Vivo}

Direct hemoperfusion under heparin infusion for $2 \mathrm{~h}$ with an animal-use polymyxin B-immobilized fiber column improved mortality and hemodynamic parameters in endotoxemic dogs receiving intravenous endotoxin administration, compared with carrier fiber alone, charcoal, and ionexchange resin (Fig. 19.7) [25]. Direct hemoperfusion with an animal-use polymyxin B-immobilized fiber column has also been shown to improve hemodynamic parameters in dogs with sepsis induced by an intravenous infusion of E. coli. Blood lactate levels were improved in dogs treated with polymyxin B-immobilized fibers compared with non-treated dogs; however,
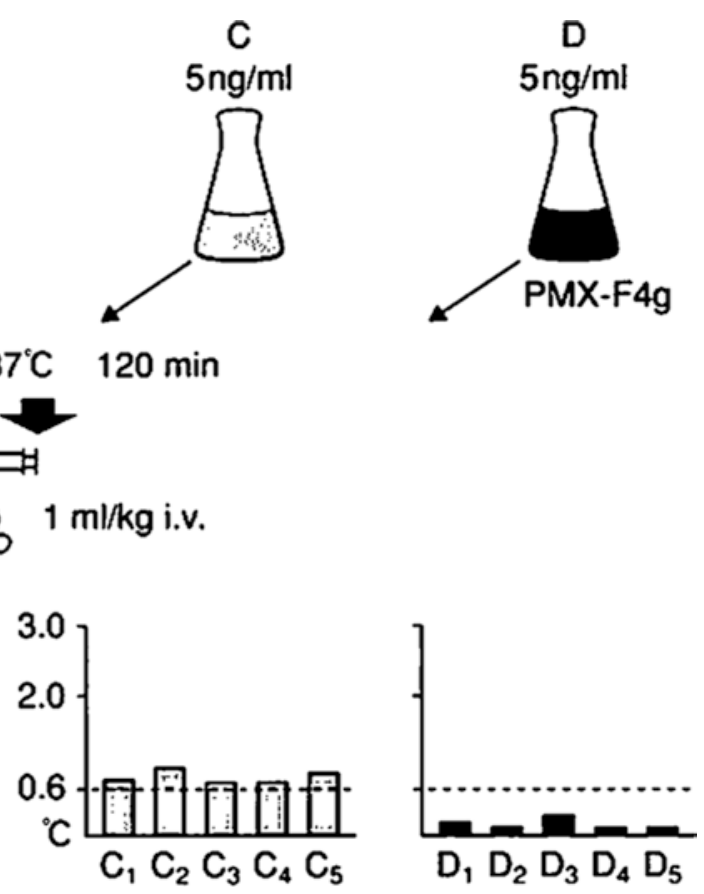

aqueous solution at $37{ }^{\circ} \mathrm{C}$ for $120 \mathrm{~min}$, and then intravenously administered to rabbits. Treatment with polymyxin B-immobilized fibers suppressed the elevation of body temperature

platelet counts were significantly lower, which is an adverse effect of direct hemoperfusion with a polymyxin B-immobilized fiber column for animal use (Fig. 19.8) [9].

\subsection{Toraymyxin, a Polymyxin B-immobilized Fiber Column for Human Use}

\subsubsection{Physical Structure of Toraymyxin Cartridge}

The Toraymyxin cartridge comprises a plastic case containing a knitted roll of polymyxin B-immobilized fiber fabric for human use. The Toraymyxin cartridge is sterilized via autoclave and filled with physiological saline. The use of a thin, fibrous carrier produces a hemoadsorption cartridge with a large surface area that does not cause a large pressure drop in the blood flow compartment (Fig. 19.2). Flow through the 

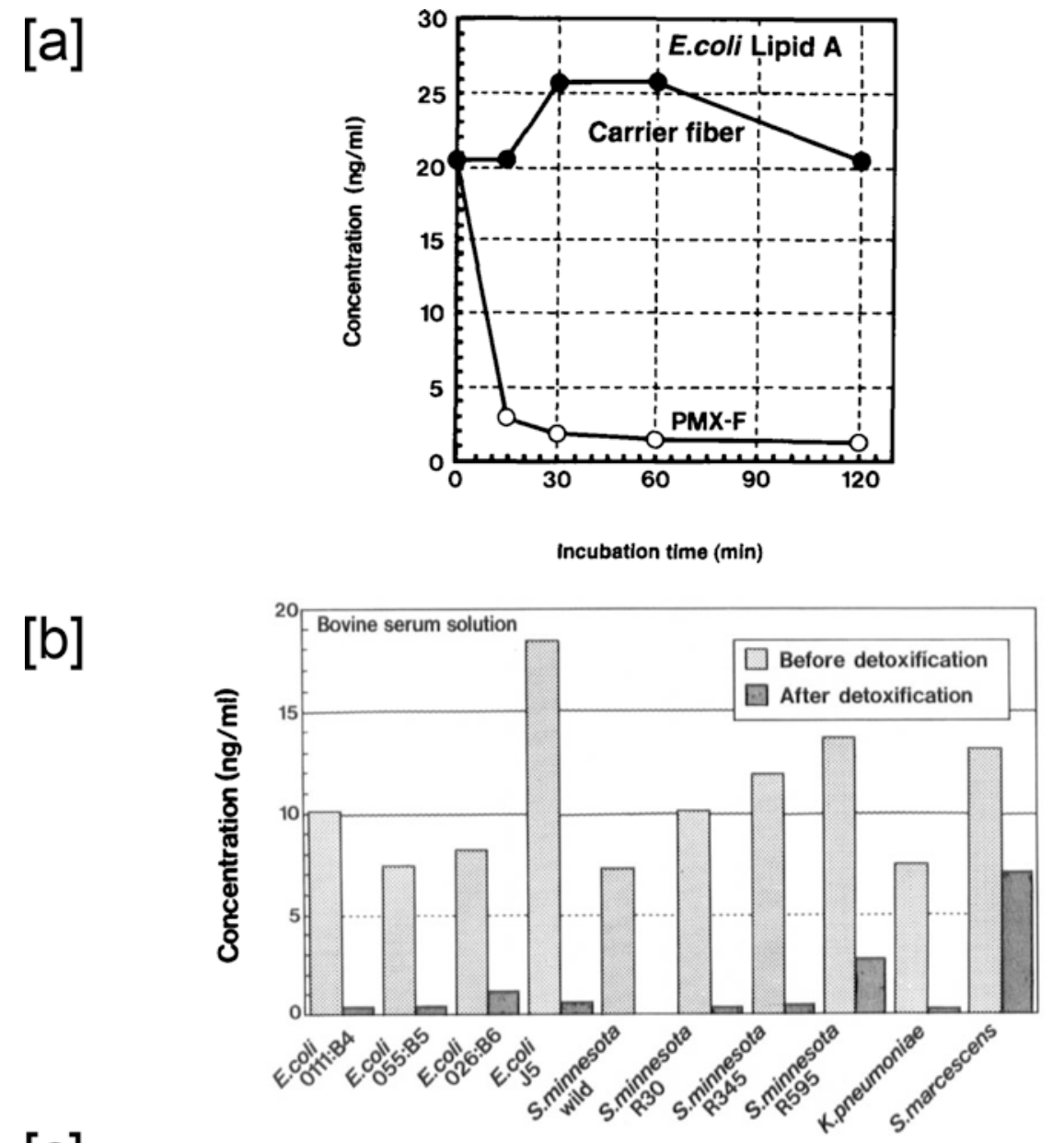

[c]

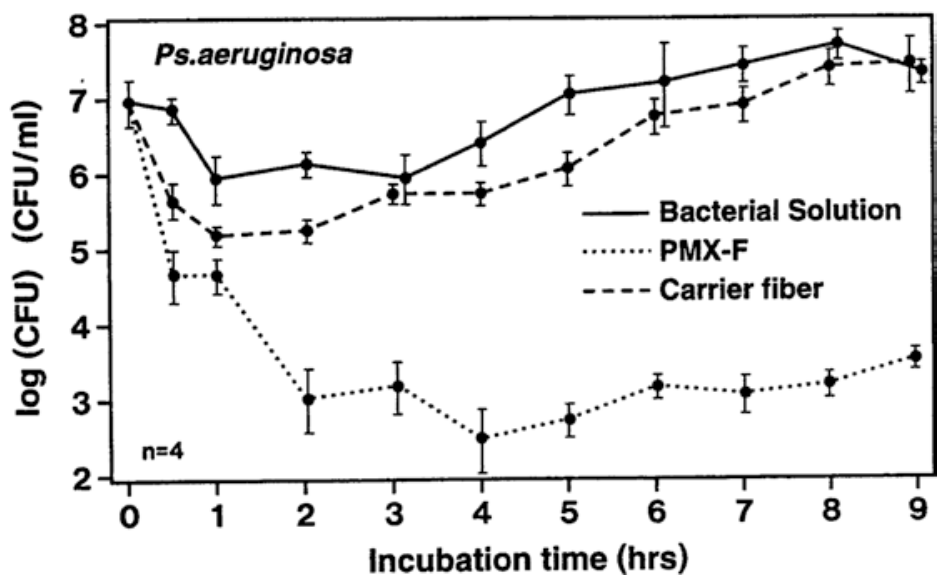

Fig. 19.6 The endotoxin adsorption ability and bactericidal activity of polymyxin B-immobilized fibers. (Reproduced from Shoji et al. [24])

(a) Alteration in lipid A concentration by polymyxin B-immobilized fibers

Polymyxin B-immobilized fibers (PMX-F) were incubated with a bovine serum solution containing $20 \mathrm{ng} / \mathrm{mL}$ of synthetic Escherichia coli lipid A with continuous stirring. Lipid A concentration was measured via limulus amebocyte lysate assay (b) Detoxification of endotoxin extracted from different species or strains of Gram-negative bacteria by use of polymyxin B-immobilized fibers

(c) Bactericidal activity of polymyxin B-immobilized fibers against Pseudomonas aeruginosa

Polymyxin B-immobilized fibers (PMX-F) or carrier fibers alone were incubated with a solution of Pseudomonas aeruginosa for $9 \mathrm{~h}$ and residual bacterial numbers were assessed 
Fig. 19.7 Endotoxin adsorption by polymyxin

B-immobilized fibers in an endotoxemia dog model. (Reproduced from Kodama et al. [25])

(a) Schematic of the experimental model: endotoxin was infused intravenously to dogs. Extracorporeal direct-hemoperfusion with a polymyxin B-immobilized fiber (PMX-F) column or control column was initiated

(b) Survival rates after PMX-F treatment were compared with those after treatment with a PMX-F column, or a charcoal, resin, or carrier fiber column [a]

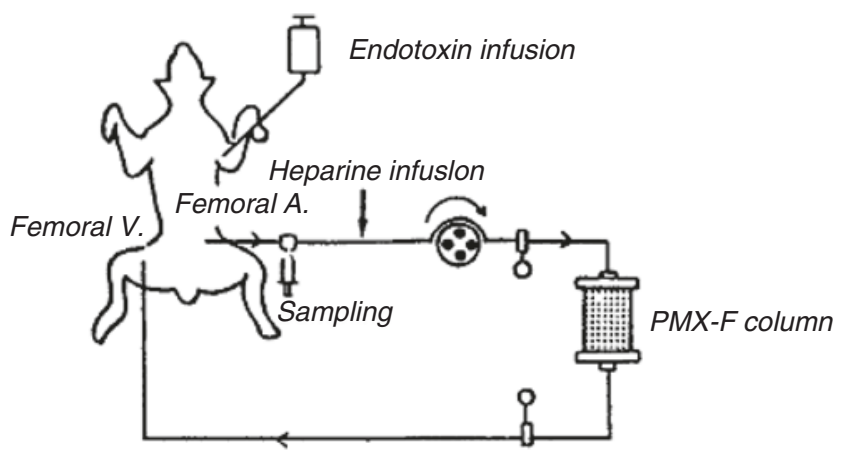

[b]

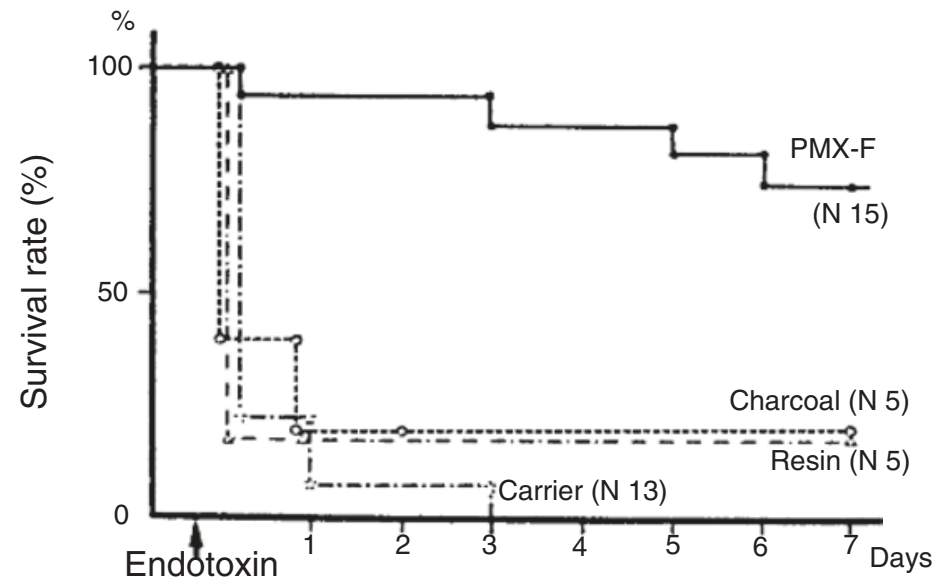

Toraymyxin column is unidirectional and moves radially from the center to the outside of the roll of polymyxin B-immobilized fiber fabric, which improves adsorption capacity by ensuring a homogeneous distribution of blood within the column (Fig. 19.9a). Therefore, direct hemoperfusion can be performed in the clinical setting without the need for a complicated extracorporeal blood perfusion circuit.

The endotoxin adsorption capacity of the Toraymyxin column was evaluated in a bovine serum perfusion model. An endotoxin-containing bovine serum solution $(10 \mathrm{ng} / \mathrm{mL}$ of endotoxin, total $1.5 \mathrm{~L}$ ) was perfused through the Toraymyxin cartridge at a flow rate of $100 \mathrm{~mL} / \mathrm{min}$, and the change in endotoxin level was monitored using an LAL assay. Endotoxin concentration reached equilibrium after $2 \mathrm{~h}$ of perfusion (Fig. 19.9b) [11].

\subsubsection{Toraymyxin as a Medical Device}

There are three types of Toraymyxin columns currently available for clinical use in Japan: PMX-20R ${ }^{\circledR} \quad$ (volume: $135 \mathrm{~mL}$ ), PMX-05R ${ }^{\circledR}$ (40 mL), and PMX-01R ${ }^{\circledR}$ (8 mL) (Fig. 19.10a). The first Toraymyxin column released was PMX20R, which was developed for the treatment of adults with septic shock [11]. The second column released was PMX-05R, which was developed for use in pediatric or elderly patients with smaller circulating blood volumes or a body weight less than $40 \mathrm{~kg}$ [26]. Several reports in Japanese literature have shown acceptable results by using PMX-05R in pediatric or elderly patients with sepsis. In 2011, PMX-01R was released for use in newborn or premature babies, and there are case reports describing the clinical use of PMX- 
[a]

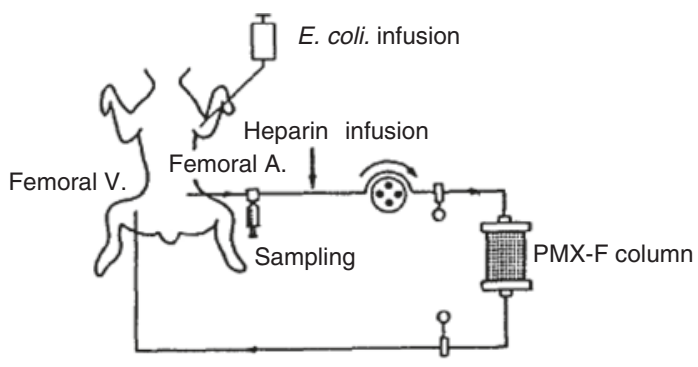

[c]

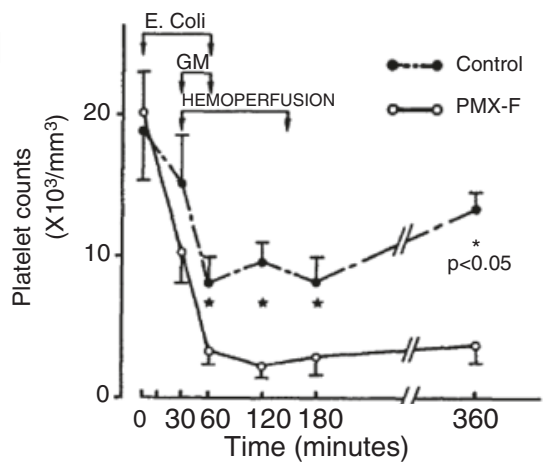

Fig. 19.8 Efficacy of polymyxin B-immobilized fibers in a dog bacterial infusion model. (Reproduced from Hanasawa et al. [9])

(a) Schematic of the experimental model: the solution containing Escherichia coli was infused intravenously to dogs. Extracorporeal direct-hemoperfusion with a polymyxin B-immobilized fiber (PMX-F) column or control column was initiated

$01 \mathrm{R}$ in patients with a body weight less than $1000 \mathrm{~g}$ with severe sepsis $[27,28]$.

\subsubsection{Use of the Toraymyxin Column in Hemoperfusion}

Prior to use, Toraymyxin columns must be rinsed with physiological saline and then primed. For example, a PMX-20R column must be washed out with $4 \mathrm{~L}$ of physiological saline and then primed with $1 \mathrm{~L}$ of physiological saline containing $20 \mathrm{mg}$ of nafamostat mesilate or $1000 \mathrm{U}$ of unfractionated heparin. For patients in an extremely critical condition or with an extremely low blood pressure, an albumin solution contain- [b]

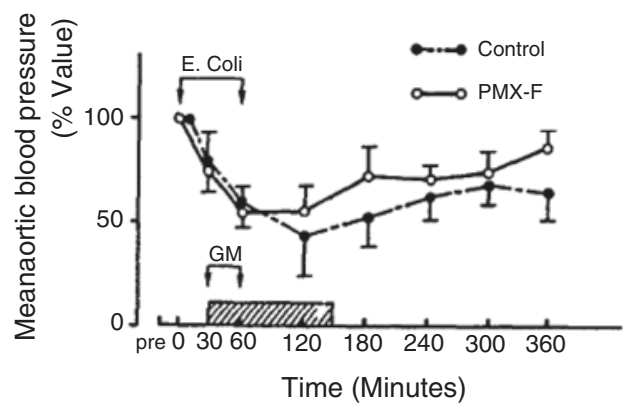

[d]

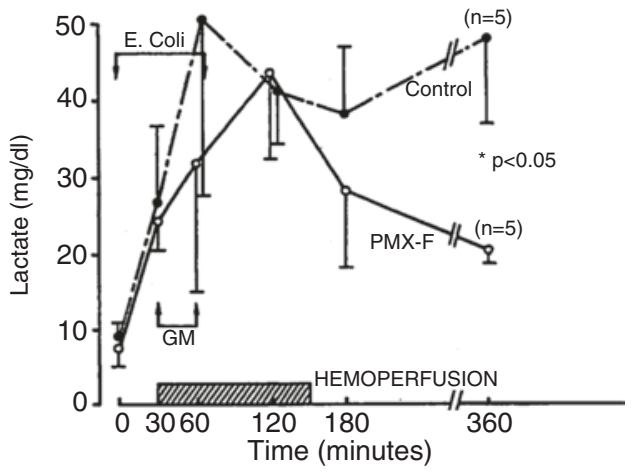

(b) Alteration in mean aortic blood pressure: mean aortic blood pressure after PMX-F treatment increased compared to that after treatment with the control column

(c) Alteration in platelet counts: platelet count significantly decreased following PMX-F treatment compared that following treatment with the control column

(d) Alteration in blood lactate levels: blood lactate levels significantly decreased following PMX-F treatment compared to those following treatment with the control column

ing anticoagulation agents may be used as the final priming solution.

Vascular access for hemoperfusion is usually via a central vein (internal cervical vein, subclavian vein, or femoral vein). The standard blood flow rate through the Toraymyxin column depends on the size of the column: $80-120 \mathrm{~mL} /$ min for PMX-20R, 20-40 mL/min for PMX05R, and 8-12 mL/min for PMX-01R. An anticoagulant $(20-30 \mathrm{mg} / \mathrm{h}$ nafamostat mesilate or bolus 40-60 U/kg and 40-60 U/kg/h unfractionated heparin, depends on patients' coagulation condition) should also be administered to prevent coagulation within the blood circuit (Fig. 19.10b). Since coagulation dysfunction is common in patients with septic shock, the short half-lives of 
Fig. 19.9 The inside structure and endotoxin adsorption capacity of the Toraymyxin cartridge

(a) Structure of a Toraymyxin cartridge and schematic of the blood flow inside the adsorption cartridge. (Provided by Toray Medical Co., Ltd.)

(b) Endotoxin adsorption capacity of the Toraymyxin cartridge compared with the carrier fiber cartridge alone. Bovine serum solution containing $10 \mathrm{ng} / \mathrm{mL}$ of endotoxin was circulated through the Toraymyxin and carrier fiber cartridges. (Reproduced from Shoji et al. [11]) [a]
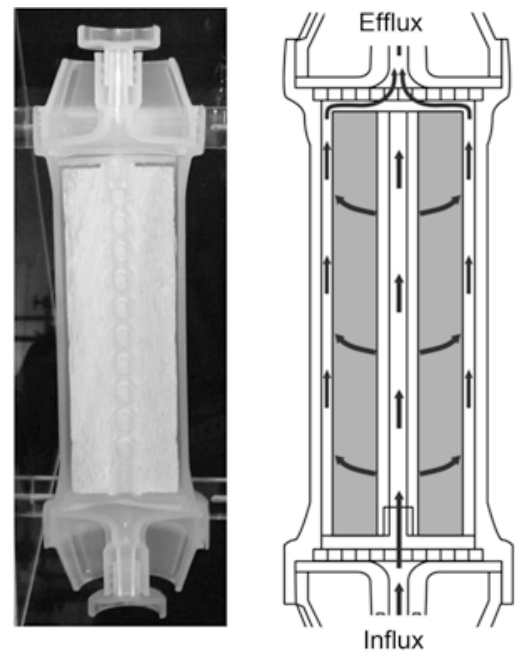

[b]

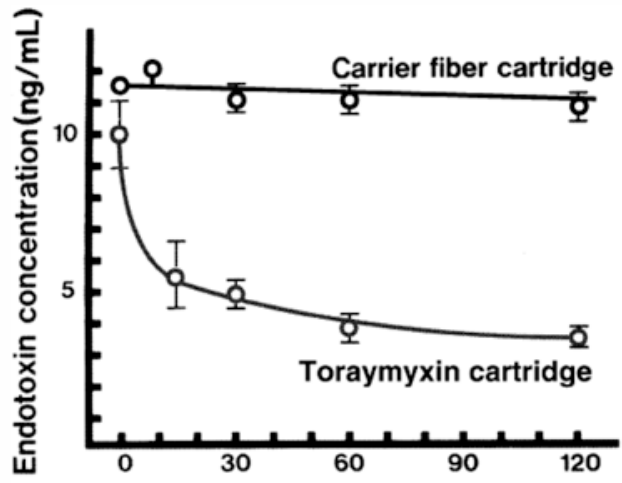

Circulation time $(\mathrm{min})$ these regional anticoagulants in the blood mean that they are safe for use during hemoperfusion. Nafamostat mesilate is the most commonly used anticoagulant for this purpose in Japan.

Since 1994, Toraymyxin has been used in more than 100,000 cases in Japan. Despite adverse effects such as thrombocytopenia or hypotension being reported, their incidence is rare, and there have been no reports of any serious adverse effects [11].

\subsection{Mechanism of Action of PMX Therapy}

\subsubsection{Removal of Endotoxin}

The Toraymyxin column was originally designed to specifically adsorb endotoxin. Plasma endo- toxin levels significantly decrease immediately after PMX treatment compared with pre-treatment levels [10]. By using data from a multicenter study, Tani reported that PMX treatment significantly reduced plasma endotoxin concentrations from $83.7 \pm 26.7 \mathrm{pg} / \mathrm{mL}$ to $56.4 \pm 27.9 \mathrm{pg} / \mathrm{mL}$ after $2 \mathrm{~h}$ of direct hemoperfusion with a Toraymyxin column [29]. Although almost all of the studies conducted so far have demonstrated a significant decrease in plasma endotoxin levels following PMX treatment, a recent RCT failed to show such an effect [12]. However, this could be due to the use of the LAL assay, which is prone to contamination and lacks precision and accuracy resulting in both false positive and false negative results [30]. Indeed, our previous study showed that sensitivity for the detection of endotoxin in the turbidimetric LAL assay was very low (26.9\%; 14 of 52 patients) in patients with severe sepsis and sep- 
[a]

PMX-20R

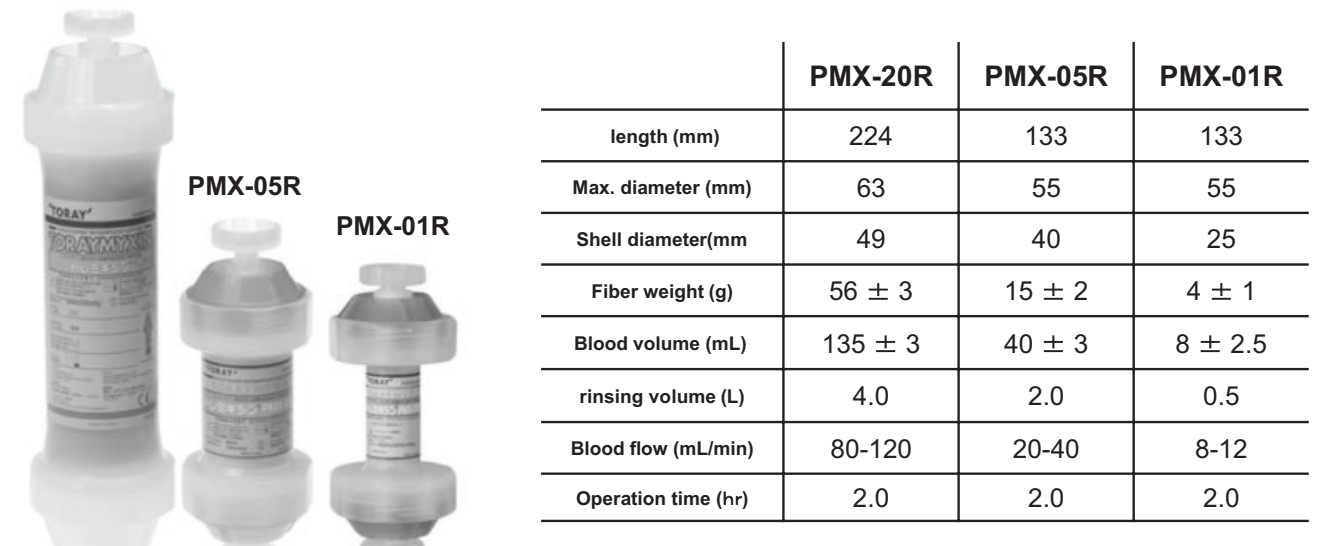

[b]

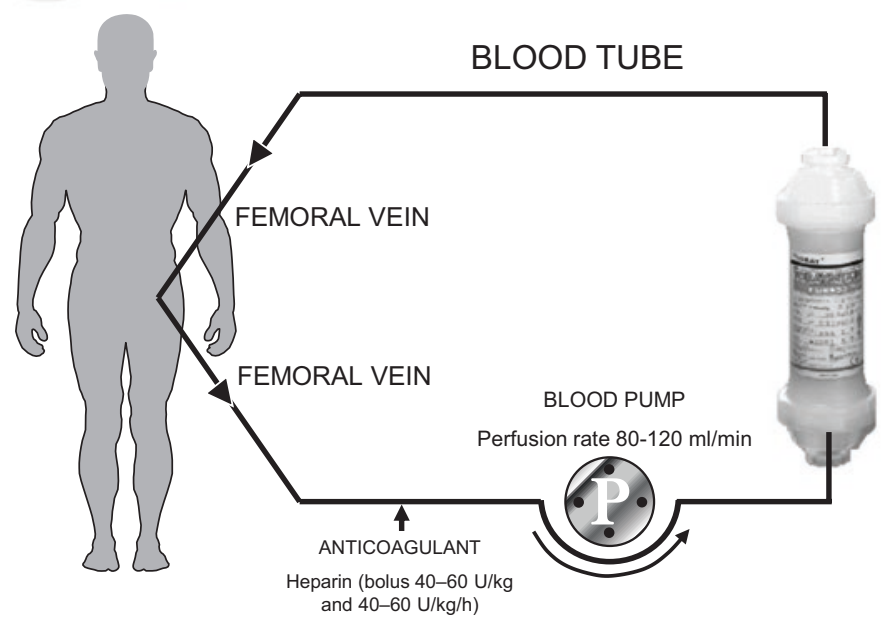

Fig. 19.10 The specifications of the Toraymyxin cartridge

(a) Overview of the three Toraymyxin cartridges currently available for clinical use. (Provided by Toray Medical Co., Ltd.)

tic shock who required PMX treatment [31]. A new method for the detection and quantitation of endotoxin is therefore immediately needed.

Recently, Romaschin developed a rapid assay, called the endotoxin activity assay (EAA), that detects endotoxin in whole blood by using autologous neutrophil-dependent chemiluminescence [32]. The EEA has been approved by the United States Food and Drug Administration as a clinical method for the diagnosis of endotoxemia, and was used in the EUPHRATES trial (see Sect. 19.5.1) [33]. (b) Schematic of direct hemoperfusion with a Toraymyxin cartridge. (Provided by Toray Medical Co., Ltd.)

A rapid LAL assay for detecting endotoxin by using a laser light-scattering particle-counting method called endotoxin scattering photometry (ESP) has also been developed. Endotoxin binds to and activates factor $\mathrm{C}$, a clotting enzyme, early in the LAL cascade, which ultimately results in the production of the insoluble protein coagulin. Coagulin spontaneously forms a polymer, and it is this polymer formation that is detected in the turbidimetric LAL assay. Obata et al. reported that by using ESP they were able to detect coagulin particles formed under agitation of the LAL cascade, and that the transferring period of the 
LAL cascade after endotoxin stimulation depends on the concentration of endotoxin before gelation [34]. The ESP method allows for more rapid and sensitive detection of endotoxin compared with the turbidimetric LAL method.

Furthermore, ESP is able to discriminate between patients with sepsis and those with septic shock undergoing gastrointestinal emergency surgery compared with the standard turbidimetric LAL assay [35]. After undergoing PMX therapy for a longer-duration ( $>2 \mathrm{~h}$ ), the hemodynamic condition of patients with septic shock due to intra-abdominal infection improved, and the reduction in plasma endotoxin concentration could be detected via ESP but not through the standard turbidimetric method. We have demonstrated the ability of endotoxin adsorption in longer-duration PMX treatment [36]. Using the ESP method, we observed a reduction in endotoxin after passing through the Toraymyxin column even when PMX duration was greater than $2 \mathrm{~h}$. Therefore, ESP appears to be sensitive enough to detect circulating endotoxin in the plasma of patients with septic shock who require PMX therapy.

\subsubsection{Other Potential Mechanism of Action of PMX Therapy}

Although Toraymyxin was originally developed for the adsorption of circulating endotoxin from the blood, recent studies have shown other potential applications. Jaber reported that it may be possible to use Toraymyxin to remove lipoteichoic acid (LTA) from peripheral blood [47]. In an in vitro study, polymyxin B significantly suppressed LTA-induced tumor necrosis factor (TNF)- $\alpha$ production by peripheral blood mononuclear cells, suggesting that LTA may also be removed during direct hemoperfusion with PMX and that this removal may be due to binding of LTA to polymyxin B.

Other recent studies have reported a possible relationship between immune cells and the reduction in inflammatory mediators. Ono demonstrated that the expression levels of HLA-DR, a major histocompatibility complex class II cell surface receptor on monocytes, and CD16 on granulocytes, markedly decrease in patients with septic shock. However, PMX treatment had beneficial effects by increasing leukocyte expression of these surface antigens [37]. The number of $\mathrm{CD}_{16}{ }^{+} \mathrm{CD} 14^{+}$monocytes and the expression level of monocytic toll-like receptor 4 dramatically increase in patients with severe infection, and recent studies have shown that PMX treatment is effective in reducing both of these in patients with septic shock [38]. Moreover, Ono demonstrated that the removal of surplus circulating $\mathrm{CD}^{+}{ }^{+} \mathrm{CD} 25^{+} \mathrm{Foxp}^{+}$regulatory $\mathrm{T}$ cells in patients with septic shock via hemoperfusion with Toraymyxin might represent a novel strategy for inducing recovery from sepsis-associated immunosuppression [39]. Tsuzuki have demonstrated immediate inhibition of NF- $\kappa \mathrm{B}$ binding activity and suppression of TNF- $\alpha$ secretion after endotoxin neutralization with polymyxin B regardless of whether the peripheral blood mononuclear cells were already producing TNF- $\alpha$ or not [40]. Reducing the number of circulating monocytes or modulating their function may contribute to improving pro-inflammatory responses following PMX treatment.

Anandamide and 2-arachidonyl glyceride are endogenous cannabinoids that are released by activated macrophages and platelets during endotoxic shock, and play a crucial role in the induction of shock-related hypotension [41]. Wang showed that anandamide was efficiently adsorbed by a polymyxin B-immobilized bead column in vitro [42]. Therefore, the adsorption of cannabinoids by polymyxin B may be an important mechanism for improving hemodynamic dysfunction following PMX treatment.

Macrophage migration inhibitory factor (MIF) is constitutively expressed by monocytes, macrophages, T cells, B cells, endocrine cells, and epithelial cells. Microbial toxins and cytokines are powerful inducers of MIF release by immune cells, and up-regulation of MIF expression during the course of inflammatory and infectious diseases plays an important role in the pathogenesis of sepsis and septic shock [43]. In a recent study, we demonstrated that Toraymyxin directly adsorbed MIF in vitro (unpublished data). 
High-mobility group box 1 protein (HMGB1), which is a protein previously known only as a nuclear transcription factor, is now implicated as a mediator of delayed endotoxin lethality and systemic inflammation [44]. An experimental study demonstrated that serum levels of HMGB1 were lower in PMX-treated patients than in controls [45]. The reduction in circulating HMGB1 level may also contribute to the beneficial effects of PMX treatment in patients with sepsis [46-48].

Further studies are required to investigate the clinical efficacy of PMX treatment and elucidate the precise mechanism. Nevertheless, in Japan, PMX treatment is a widely accepted method to improve the condition of patients with septic shock, and this useful clinical device is gaining acceptance as its use becomes more widespread.

\subsection{Clinical Use of PMX Therapy}

\subsubsection{PMX Therapy for the Treatment of Sepsis}

The first clinical report from a phase I study of PMX therapy for the treatment of sepsis was published in 1994 [10]. Sixteen patients with septic multiple organ failure were treated with direct hemoperfusion with a Toraymyxin column over $2 \mathrm{~h}$. PMX treatment significantly decreased the endotoxin level from 76 to $21 \mathrm{pg} / \mathrm{mL}$ (Fig. 19.11a). Furthermore, the patients' hyperdynamic state (in terms of cardiac index), which is characteristic of endotoxic shock, returned to normal after treatment (Fig. 19.11b) [10].

The results of the first prospective, multicenter, observational clinical trial of Toraymyxin for the treatment of patients with sepsis in Japan were published in 1998 [29]. The survival rate was significantly higher in the treatment (PMX) group (54.0\%) compared with the control group who received standard therapies $(36.4 \%)$. In the treatment group, the mean plasma endotoxin concentration was significantly lowered from $83.7 \mathrm{pg} / \mathrm{mL}$ before treatment to $56.4 \mathrm{pg} / \mathrm{mL}$ immediately after treatment. The day after treatment, the mean plasma endotoxin concentration was found to be $28.5 \mathrm{pg} / \mathrm{mL}$. Post treatment, the mean plasma endotoxin concentration was lower in those who survived $(18.8 \mathrm{pg} / \mathrm{mL})$ compared with those who died $(88 \mathrm{pg} / \mathrm{mL})$. Other cardiac function parameters also improved after treatment.

Nemoto demonstrated that the rate of overall survival in patients with sepsis significantly improved after PMX treatment compared with standard therapies without PMX (41\% vs. $11 \%$ ) in a RCT of 98 patients with sepsis in Japan [31]. PMX improved the rate of survival in patients with an APACHE II (Acute Physiology and Chronic Health Evaluation II) score $<30$, but not in those with scores $>30$, demonstrating that Toraymyxin treatment is most effective at improving patient outcome when applied during the early stages of sepsis [49].

In 1998, Toraymyxin received CE mark approval in Europe. In 2005, Vincent et al. published the results of a multicenter, open-label, pilot, randomized controlled study conducted in the intensive care units of six academic medical centers in Europe, which was the first RCT of Toraymyxin conducted outside of Japan [11]. Thirty-six postoperative patients with severe sepsis or septic shock secondary to intra-abdominal infection were randomized to either PMX treatment for $2 \mathrm{~h}(n=17)$ or standard therapy $(n=19)$. PMX treatment was well tolerated and no significant adverse effects were observed. Patients treated with PMX showed significant increases in cardiac index $(P=0.012$ and 0.032 at days 1 and 2 , respectively), left ventricular stroke work index $(P=0.015$ at day 2$)$, and oxygen delivery index $(P=0.007$ at day 2$)$ compared with controls. Furthermore, the need for continuous renal replacement therapy after study entry was significantly reduced in the PMX group $(P=0.043)$. There were no statistically significant differences in endotoxin and interleukin six levels, and organ dysfunction as assessed by the Sequential Organ Failure Assessment (SOFA) score, or 28-day mortality. Note that sepsis in the patients enrolled in this study was likely too severe to find statistical significance in the mortality outcome. Together, these results show that PMX treatment 
[a]

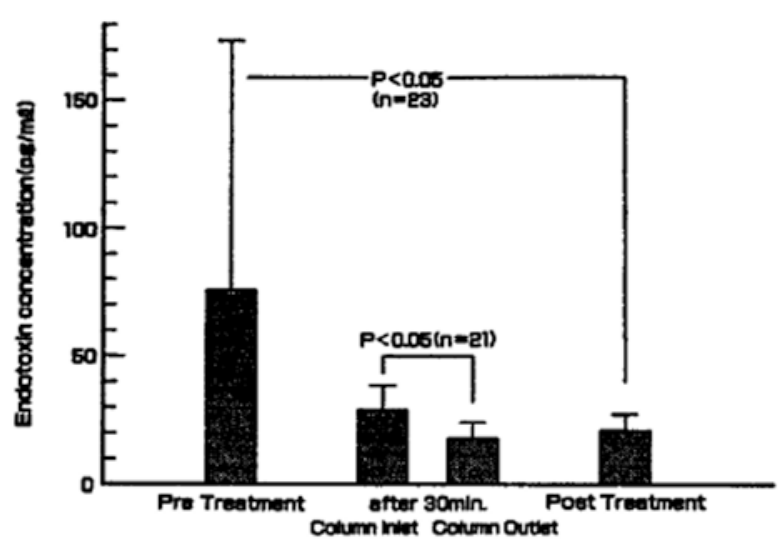

[b]

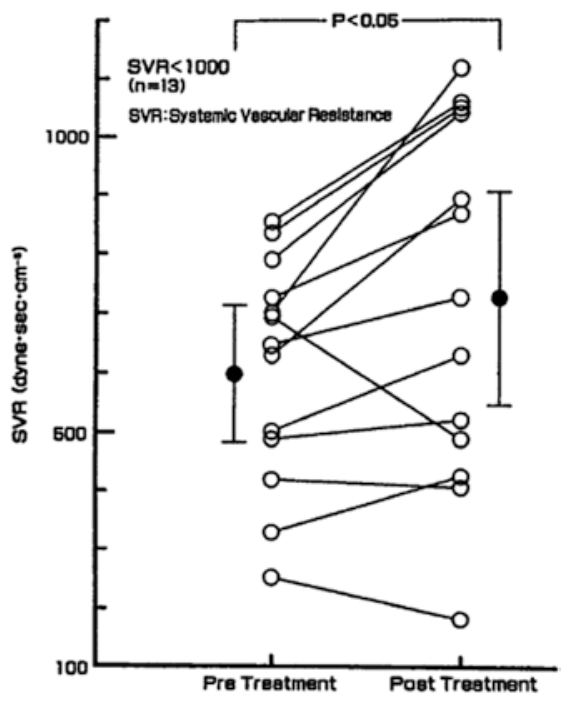

Fig. 19.11 Results from the first clinical trial of Toraymyxin in Japan

(a) Endotoxin concentrations before and after Toraymyxin treatment

is safe and that it improves cardiac and renal dysfunction due to sepsis or septic shock.

In 2007, Cruz et al. published a systematic review of the effectiveness of Toraymyxin for the treatment of sepsis [12]. They included 28 publications that reported at least one of the specified outcome measures for PMX therapy. In this meta-analysis of 1425 patients (PMX therapy, 978 patients; conventional therapy, 447 patients), PMX therapy was associated with a significantly lower risk of mortality compared with conventional therapy (PMX, 33.5\% vs. conventional treatment, $66.5 \%$; risk ratio, 0.53 ; $95 \%$ CI, $0.43-$ $0.65)$. A $33-80 \%$ reduction in plasma endotoxin levels compared to pre-treatment levels was also observed. The large decrease in mortality was associated with an improvement in hemodynamic condition: after PMX therapy, mean arterial pressure significantly increased by $19 \mathrm{mmHg}$ (mean increase, 26\%; range, 14-42\%), and dopamine/ dobutamine dose was decreased by $1.8 \mu \mathrm{g} / \mathrm{kg} /$ min. Improvement in pulmonary function was also demonstrated: the mean ratio of partial pressure arterial oxygen to fraction of inspired oxygen $\left(\mathrm{PaO}_{2} / \mathrm{FiO}_{2}\right)$ increased by 32 units $(95 \% \mathrm{CI}$, 23-41 units; $P<0.001$ ) (Fig. 19.12). (b) Systemic vascular resistance (SVR) before and after Toraymyxin treatment. Toraymyxin treatment decreased the concentration of endotoxin in the blood and improved hemodynamic status in patients with severe sepsis and septic shock. (Reproduced from Aoki et al. [10])

Cruz et al. have also published the results of the EUPHAS (Early Use of Polymyxin B Hemoperfusion in Abdominal Sepsis) trial, which was a prospective, multicenter, RCT conducted at the intensive care units of ten Italian tertiary care hospitals in 2009 [14]. Sixty-four patients with severe sepsis or septic shock who had undergone emergency surgery for intra-abdominal infection were enrolled in this study. Patients were randomized within $6 \mathrm{~h}$ after open abdominal surgery to either conventional therapy $(n=30)$ or conventional therapy plus two sessions of $2 \mathrm{~h}$ PMX with an interval of $24 \mathrm{~h}$ between sessions $(n=34)$. Twenty-eight-day mortality was significantly improved in the PMX group (32\%; 11/ 34 patients) compared with that in the conventional therapy group (53\%; 16/30 patients). In the PMX group, mean arterial pressure significantly increased from $76 \mathrm{mmHg}$ (before treatment) to $84 \mathrm{mmHg}$ (72 h after treatment; $P=0.001)$ and vasopressor requirement (measured as inotropic score) significantly decreased from 29.9 (before treatment) to 6.8 ( $72 \mathrm{~h}$ after treatment; $P<0.001$ ). In contrast, in the conventional therapy group, mean arterial pressure (before, $74 \mathrm{mmHg} ; 72 \mathrm{~h}$ after, $77 \mathrm{mmHg} ; P=0.37$ ) and inotropic score 


\section{Increasing}

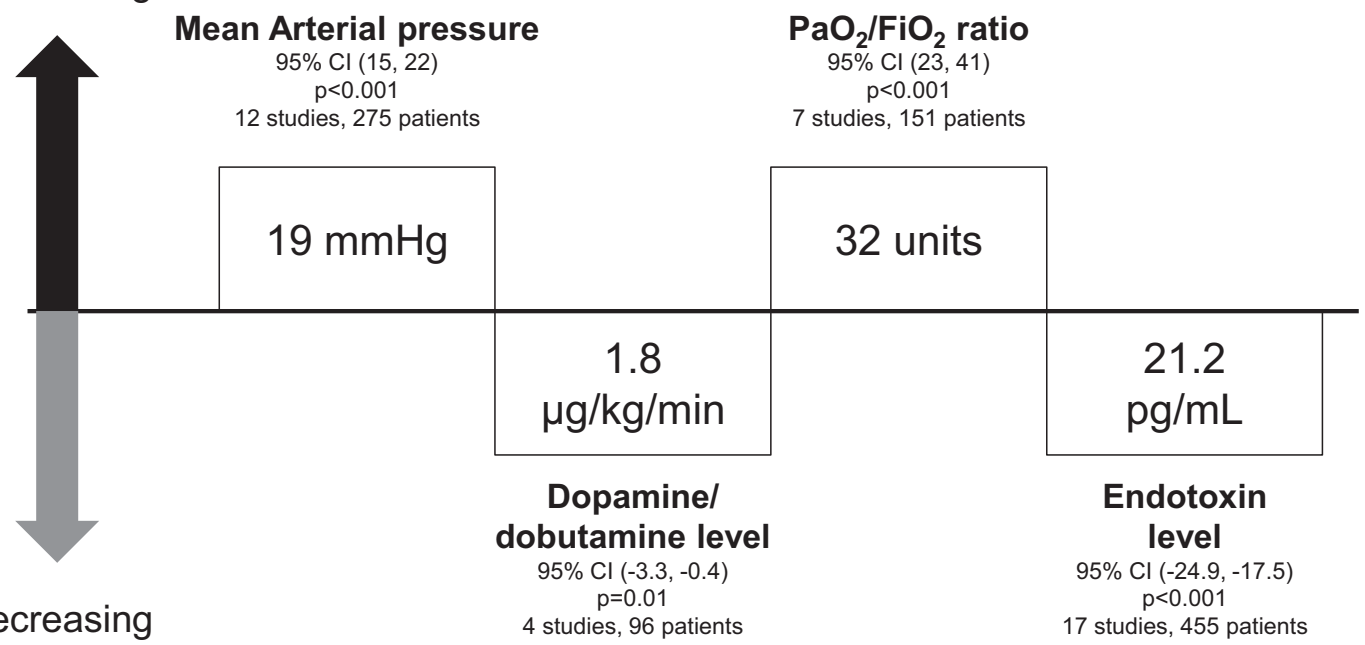

Fig. 19.12 Main results of a meta-analysis of Toraymyxin treatment. (Provided by Toray Medical Co., Ltd.) $M A P$ mean arterial pressure, $\mathrm{PaO}_{2} / \mathrm{FIO}_{2}$ ratio of partial pressure arterial oxygen and fraction of inspired oxygen, CI confidence interval

induced septic shock from abdominal infections were enrolled and the primary end point of the study was 28-day mortality. This multicenter randomized controlled study demonstrated a nonsignificant increase in mortality and no improvement in organ failure with PMX treatment compared to conventional treatment of peritonitis-induced septic shock. However, Antonelli et al. pointed out that major differences in mortality and completion rates of two scheduled sessions of PMX compared to the EUPHAS study may jeopardize their comparability, suggesting that any definitive conclusion be put on hold [50]. The 28-day mortality rate recorded in both groups was significantly lower than that reported in larger studies (between $32.7 \%$ and $53 \%$ for similar patient cohorts) [51-53]. Furthermore, only 81 of the 119 treated patients completed the two scheduled sessions of PMX. In the previously published EUPHAS study, all patients enrolled had completed the two planned sessions of PMX, and a significantly higher mortality rate was recorded in the control group [50].

The EUPHRATES trial (Evaluating the Use of Polymyxin B Hemoperfusion in a Randomized Controlled Trial of Adults Treated for Endotoxemia and Septic Shock), which is ongo- 
ing in the USA and Canada [33], is a trial designed to address the criticisms of previous studies. Circulating endotoxin levels in patients with septic shock were evaluated by using a novel endotoxin detection method called the endotoxin activity assay (EAA). A total of 432 patients with septic shock and high EAA activity $(>0.6)$ were enrolled and randomized to either PMX or conventional treatment. Dellinger et al. reported that the primary endpoint for mortality rate $(44.3 \%$ in placebo group and $43.75 \%$ in PMX group) was not met in the full intention-to-treat population. Their interim results showed that the mortality rate in the per protocol population $(36.9 \%$ in placebo group and $31.9 \%$ in PMX group) was $5 \%$ in favor of PMX treatment $(P=0.407)$. Interestingly, the final results of the EUPHRATES trial did not show reduced mortality at 28 days in patients with PMX treatment (ClinicalTrials.gov Identifier: NCT01046669).

Direct hemoperfusion sessions with Toraymyxin column usually last $2 \mathrm{~h}$; however, the obvious clinical efficacy of longer-duration PMX ( $>6 \mathrm{~h})$ in such cases has been reported [36, 54-56]. No adverse effects, such as thrombocytopenia, have been reported with longer duration Toraymyxin treatment $[36,54]$. Further studies to clarify the suitability of longer-duration Toraymyxin treatment are expected.

The combination of PMX and continuous hemodiafiltration (CHDF) has been reported to be more beneficial for patients with septic renal dysfunction than CHDF alone. Combination treatment significantly decreases the concentration of circulating interleukin-6 and improves patient survival (Fig. 19.13a) [57]. Moreover, PMX followed by CHDF with a polymethyl methacrylate (PMMA) membrane significantly decreased the concentrations of plasminogen activator inhibitor-1, protein $\mathrm{C}$, interleukin-6, and endogenous anandamide compared with CHDF with a polyacrylonitrile membrane in patients with septic shock [58]. Therefore, the combination of PMX with PMMA-CHDF is beneficial for patients with septic shock and septic renal dysfunction. In combination therapy, the Toraymyxin column is generally placed before or after CHDF on a single circuit. However, when
CHDF cannot be interrupted, for example in patients with renal failure, the Toraymyxin circuit can be connected in parallel or in series with the CHDF circuit (Fig. 19.13b) [59].

\subsubsection{PMX Therapy for the Treatment of Acute Exacerbation of Idiopathic Pulmonary Fibrosis and Acute Respiratory Distress Syndrome}

Idiopathic pulmonary fibrosis (IPF) is one of a heterogeneous group of diffuse parenchymal lung disorders of unknown etiology. Acute exacerbation of IPF is characterized by severe worsening dyspnea and high mortality. IPF is one of the most common presentations of idiopathic interstitial pneumonia. Previous reports have suggested that PMX treatment improves oxygenation in patients with acute lung injury or acute respiratory distress syndrome (ARDS) due to severe sepsis $[60,61]$. The potential benefit of PMX therapy for the treatment of acute exacerbation of IPF has also been reported [62]. A multicenter retrospective analysis of 160 patients showed that PMX therapy improves oxygenation and survival in patients experiencing acute exacerbation of IPF [63]. A largescale, prospective, RCT in patients experiencing acute exacerbation of IPF will be conducted in the near future.

The successful treatment of severe ARDS due to influenza virus infection with PMX has been reported recently. Yokoyama et al. reported a case of severe ARDS caused by novel swine-origin influenza virus (A/H1N1pdm) [64]. The patient underwent PMX, after which her hypoxemia improved and she survived. This is the first report of severe, life-threatening ARDS due to a novel influenza virus in which PMX was beneficial. Yatera et al. reported a case of ARDS due to influenza A infection that was successfully treated with PMX [65]. Kudo et al. has reported cases of severe pneumonia due to highly pathogenic avian influenza A (H5N1) in Vietnam successfully treated with CHDF coupled with Toraymyxin hemoperfusion, suggesting that it is 
Fig. 19.13 Use of

Toraymyxin in combination with continuous hemodiafiltration

(a) Survival of patients receiving Toraymyxin therapy in combination with continuous hemodiafiltration. (Reproduced from Suzuki et al. [57]) The use of this combination significantly improved survival rate in patients with sepsis and acute renal failure

(b) Schematic of the combination of direct hemoperfusion with Toraymyxin and continuous hemodiafiltration in a series-parallel circuit [a]
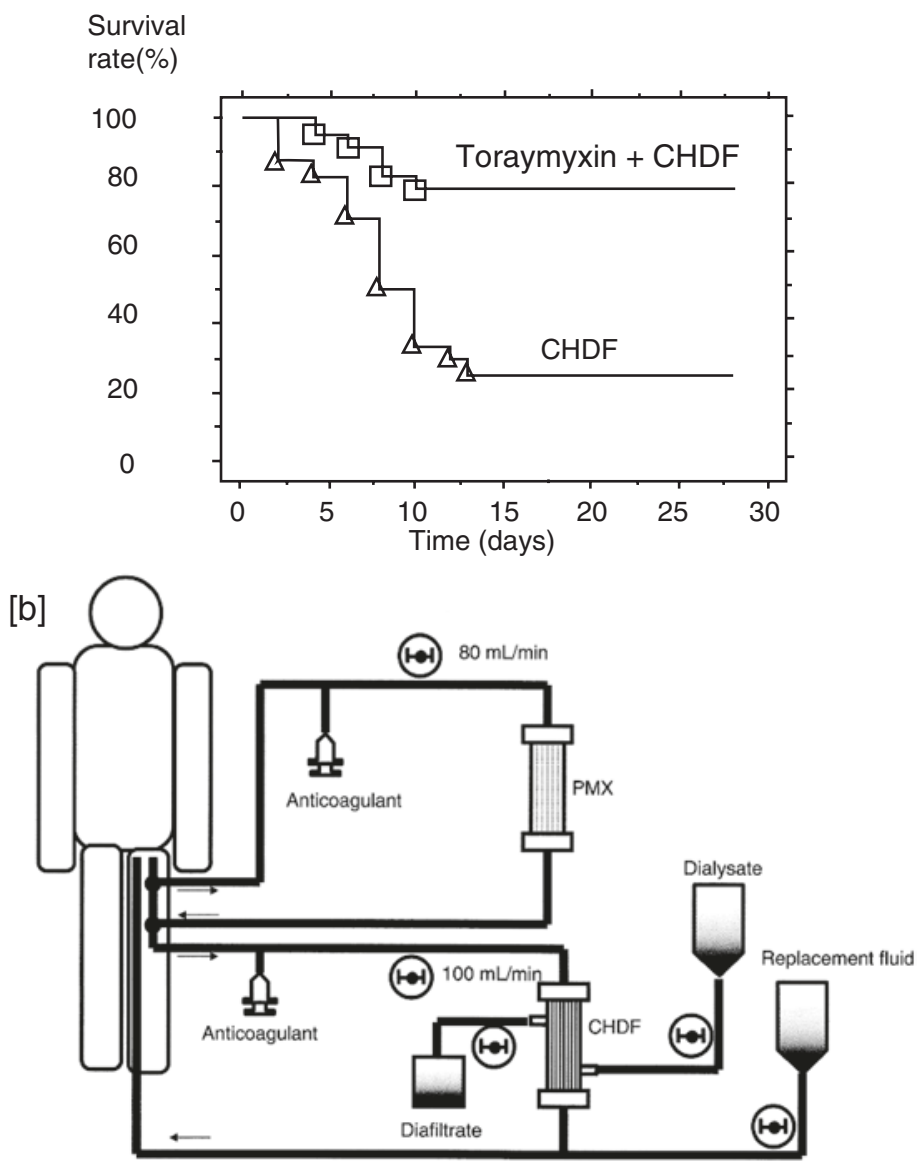

an effective candidate treatment for ARDS due to the $\mathrm{H} 5 \mathrm{~N} 1$ virus if applied early in the disease [66].

These data show that new indications for PMX treatment in the field of pulmonary medicine will likely be available in the near future.

\section{References}

1. Hurley JC, Guidet B, Offenstadt G, Maury E (2012) Endotoxemia and mortality prediction in ICU and other settings: underlying risk and co-detection of Gram-negative bacteremia are confounders. J Crit Care 16(4):R148. https://doi.org/10.1186/cc11462

2. Anspach FB (2001) Endotoxin removal by affinity sorbents. J Biochem Biophys Methods 49(1-3):665-681

3. Nolan JP, Ali MV (1972) Effect of cholestyramine on endotoxin toxicity and absorption. Am J Dig Dis 17(2):161-166
4. Cohen J, Aslam M, Pusey CD, Ryan CJ (1987) Protection from endotoxemia: a rat model of plasmapheresis and specific adsorption with polymyxin B. J Infect Dis 155(4):690-695

5. Umeda M, Niwa M, Yamagami S, Kishimot $T$, Maekawa M, Sawada Y (1990) Novel endotoxin adsorbing materials, polymyxin-sepharose and polyporous polyethylene membrane for removal of endotoxin from dialysis systems. Biomater Artif Cells Artif Organs 18(4):491-497

6. Mitzner S, Schneidewind J, Falkenhagen D, Loth F, Klinkmann H (1993) Extracorporeal endotoxin removal by immobilized polyethylenimine. Artif Organs 17(9):775-781

7. Zimmermann M, Busch K, Kuhn S, Zeppezauer M (1999) Endotoxin adsorbent based on immobilized human serum albumin. Clin Chem Lab Med 37(3):373-379. https://doi.org/10.1515/ CCLM.1999.062

8. Hanasawa K, Tani T, Oka T, Yoshioka T, Aoki H, Endo Y, Kodama M (1988) Selective removal of endotoxin from the blood by extracorporeal hemoperfusion with 
polymyxin B-immobilized fiber. Prog Clin Biol Res 264:337-341

9. Hanasawa K, Tani T, Kodama M (1989) New approach to endotoxic and septic shock by means of polymyxin B-immobilized fiber. Surg Gynecol Obstet 168(4):323-331

10. Aoki H, Kodama M, Tani T, Hanasawa K (1994) Treatment of sepsis by extracorporeal elimination of endotoxin using polymyxin B-immobilized fiber. Am J Surg 167(4):412-417

11. Shoji H (2003) Extracorporeal endotoxin removal for the treatment of sepsis: endotoxin adsorption cartridge (Toraymyxin). Ther Apher Dial 7(1):108-114

12. Vincent JL, Laterre PF, Cohen J, Burchardi H, Bruining H, Lerma FA, Wittebole X, De Backer D, Brett S, Marzo D, Nakamura H, John S (2005) A pilot-controlled study of a polymyxin B-immobilized hemoperfusion cartridge in patients with severe sepsis secondary to intra-abdominal infection. Shock 23(5):400-405

13. Cruz DN, Perazella MA, Bellomo R, de Cal M, Polanco N, Corradi V, Lentini P, Nalesso F, Ueno T, Ranieri VM, Ronco C (2007) Effectiveness of polymyxin B-immobilized fiber column in sepsis: a systematic review. Crit Care 11(2):R47. https://doi. org/10.1186/cc5780

14. Cruz DN, Antonelli M, Fumagalli R, Foltran F, Brienza N, Donati A, Malcangi V, Petrini F, Volta G, Bobbio Pallavicini FM, Rottoli F, Giunta F, Ronco C (2009) Early use of polymyxin B hemoperfusion in abdominal septic shock: the EUPHAS randomized controlled trial. JAMA 301(23):2445-2452. https:// doi.org/10.1001/jama.2009.856

15. Payen DM, Guilhot J, Launey Y, Lukaszewicz AC, Kaaki M, Veber B, Pottecher J, Joannes-Boyau O, Martin-Lefevre L, Jabaudon M, Mimoz O, Coudroy R, Ferrandiere M, Kipnis E, Vela C, Chevallier S, Mallat J, Robert R, ABDOMIX Group (2015) Early use of polymyxin $\mathrm{B}$ hemoperfusion in patients with septic shock due to peritonitis: a multicenter randomized control trial. Intensive Care Med 41(6):975-984. https://doi.org/10.1007/s00134-015-3751-z

16. Morrison DC, Jacobs DM (1976) Binding of polymyxin B to the lipid A portion of bacterial lipopolysaccharides. Immunochemistry 13(10):813-818

17. Corrigan JJ Jr, Kiernat JF (1979) Effect of polymyxin B sulfate on endotoxin activity in a Gram-negative septicemia model. Pediatr Res 13(1):48-51. https:// doi.org/10.1203/00006450-197901000-00011

18. Flynn PM, Shenep JL, Stokes DC, Fairclough D, Hildner WK (1987) Polymyxin B moderates acidosis and hypotension in established, experimental Gramnegative septicemia. J Infect Dis 156(5):706-712

19. Ingoldby CJ (1980) The value of polymixin B in endotoxaemia due to experimental obstructive jaundice and mesenteric ischaemia. Br J Surg 67(8):565-567

20. Lopes J, Inniss WE (1969) Electron microscopy of effect of polymyxin on Escherichia coli lipopolysaccharide. J Bacteriol 100(2):1128-1129
21. Ribi E, Anacker RL, Brown R, Haskins WT, Malmgren B, Milner KC, Rudbach JA (1966) Reaction of endotoxin and surfactants. I. Physical and biological properties of endotoxin treated with sodium deoxycholate. J Bacteriol 92(5):1493-1509

22. Bader J, Teuber M (1973) Action of polymyxin B on bacterial membranes. 1. Binding to the O-antigenic lipopolysaccharide of Salmonella typhimurium. Z Naturforsch 28(7):422-430

23. Vesentini S, Soncini M, Fiore GB, Redaelli A (2010) Mechanisms of polymyxin B endotoxin removal from extracorporeal blood flow: molecular interactions. Contrib Nephrol 167:45-54. https://doi. org/10.1159/000315918

24. Shoji H, Tani T, Hanasawa K, Kodama M (1998) Extracorporeal endotoxin removal by polymyxin B immobilized fiber cartridge: designing and antiendotoxin efficacy in the clinical application. Ther Apher 2(1):3-12

25. Kodama M, Hanasawa K, Tani T (1990) New therapeutic method against septic shock-removal of endotoxin using extracorporeal circulation. Adv Exp Med Biol 256:653-664

26. Nakamura T, Kawagoe Y, Suzuki T, Shoji H, Ueda Y, Koide H (2007) Polymyxin B-immobilized fiber hemoperfusion with the PMX-05R column in elderly patients suffering from septic shock. Am J Med Sci 334(4):244-247. https://doi.org/10.1097/ MAJ.0b013e3180a5e8d8

27. Nishizaki N, Nakagawa M, Hara S, Oda H, Kantake M, Obinata K, Uehara Y, Hiramatsu K, Shimizu T (2016) Effect of PMX-DHP for sepsis due to ESBLproducing $E$. coli in an extremely low-birthweight infant. Pediatr Int 58(5):411-414. https://doi. org/10.1111/ped.12825

28. Tokumasu H, Watabe S, Tokumasu S (2016) Effect of hemodiafiltration therapy in a low-birthweight infant with congenital sepsis. Pediatr Int 58(3):237-240. https://doi.org/10.1111/ped.12776

29. Tani T, Hanasawa K, Endo Y, Yoshioka T, Kodama M, Kaneko M, Uchiyama Y, Akizawa T, Takahasi K, Sugai K (1998) Therapeutic apheresis for septic patients with organ dysfunction: hemoperfusion using a polymyxin B-immobilized column. Artif Organs 22(12):1038-1044

30. Cohen J (2000) The detection and interpretation of endotoxaemia. Intensive Care Med 26(Suppl 1):S51-S56

31. Shimizu T, Hanasawa K, Sato K, Umeki M, Koga N, Naganuma T, Sato S, Shimonishi T, Ikeda T, Matsuno N, Ono S, Saitoh H, Satoh K, Otani Y, Endo Y, Eguchi Y, Tani T, Group PMXTS (2009) Direct hemoperfusion with polymyxin B-immobilized fiber columns improves septic hypotension and reduces inflammatory mediators in septic patients with colorectal perforation. Langenbeck's Arch Surg 394(2):303-311. https://doi.org/10.1007/s00423-008-0395-2

32. Romaschin AD, Harris DM, Ribeiro MB, Paice J, Foster DM, Walker PM, Marshall JC (1998) A rapid 
assay of endotoxin in whole blood using autologous neutrophil dependent chemiluminescence. J Immunol Methods 212(2):169-185

33. Foster D, Klein DJ, Guadagni G, Walker PM (2014) Endotoxin removal: bringing the mission to North America. Blood Purif 37(Suppl 1):14-17. https://doi. org/10.1159/000356832

34. Obata T, Nomura M, Kase Y, Sasaki H, Shirasawa Y (2008) Early detection of the Limulus amebocyte lysate reaction evoked by endotoxins. Anal Biochem 373(2):281-286. https://doi.org/10.1016/j. ab.2007.09.018

35. Shimizu T, Obata T, Sonoda H, Akabori H, Miyake T, Yamamoto H, Tabata T, Eguchi Y, Tani T (2013) Diagnostic potential of endotoxin scattering photometry for sepsis and septic shock. Shock 40(6):504-511. https://doi.org/10.1097/SHK.0000000000000056

36. Shimizu T, Obata T, Sonoda H, Akabori H, Tabata T, Eguchi Y, Endo Y, Tani T (2013) The ability of endotoxin adsorption during a longer duration of direct hemoperfusion with a polymyxin B-immobilized fiber column in patients with septic shock. Transfus Apher Sci 49(3):499-503. https://doi.org/10.1016/j. transci.2013.04.042

37. Ono S, Tsujimoto H, Matsumoto A, Ikuta S, Kinoshita M, Mochizuki H (2004) Modulation of human leukocyte antigen-DR on monocytes and CD16 on granulocytes in patients with septic shock using hemoperfusion with polymyxin B-immobilized fiber. Am J Surg 188(2):150-156. https://doi.org/10.1016/j. amjsurg.2003.12.067

38. Tsujimoto H, Ono S, Hiraki S, Majima T, Kawarabayashi N, Sugasawa H, Kinoshita M, Hiraide H, Mochizuki H (2004) Hemoperfusion with polymyxin B-immobilized fibers reduced the number of CD16+ CD14+ monocytes in patients with septic shock. J Endotoxin Res 10(4):229-237. https://doi. org/10.1179/096805104225005814

39. Ono S, Kimura A, Hiraki S, Takahata R, Tsujimoto H, Kinoshita M, Miyazaki H, Yamamoto J, Hase K, Saitoh D (2013) Removal of increased circulating CD4+CD25+Foxp3+ regulatory $\mathrm{T}$ cells in patients with septic shock using hemoperfusion with polymyxin B-immobilized fibers. Surgery 153(2):262271. https://doi.org/10.1016/j.surg.2012.06.023

40. Tsuzuki H, Tani T, Ueyama H, Kodama M (2001) Lipopolysaccharide: neutralization by polymyxin B shuts down the signaling pathway of nuclear factor kappa B in peripheral blood mononuclear cells, even during activation. J Surg Res 100(1):127-134. https:// doi.org/10.1006/jsre.2001.6227

41. Varga K, Wagner JA, Bridgen DT, Kunos G (1998) Platelet- and macrophage-derived endogenous cannabinoids are involved in endotoxin-induced hypotension. FASEB J 12(11):1035-1044

42. Wang Y, Liu Y, Sarker KP, Nakashima M, Serizawa T, Kishida A, Akashi M, Nakata M, Kitajima I, Maruyama I (2000) Polymyxin B binds to anandamide and inhibits its cytotoxic effect. FEBS Lett 470(2):151-155
43. Froidevaux C, Roger T, Martin C, Glauser MP, Calandra T (2001) Macrophage migration inhibitory factor and innate immune responses to bacterial infections. Crit Care Med 29(7 Suppl):S13-S15

44. Wang H, Yang H, Czura CJ, Sama AE, Tracey KJ (2001) HMGB1 as a late mediator of lethal systemic inflammation. Am J Respir Crit Care Med 164(10 Pt 1):1768-1773. https://doi.org/10.1164/ ajrccm.164.10.2106117

45. Hussein MH, Kato T, Sugiura T, Daoud GA, Suzuki S, Fukuda S, Sobajima H, Kato I, Togari H (2005) Effect of hemoperfusion using polymyxin B-immobilized fiber on IL-6, HMGB-1, and IFN gamma in a neonatal sepsis model. Pediatr Res 58(2):309-314. https://doi. org/10.1203/01.PDR.0000169995.25333.F7

46. Sakamoto Y, Mashiko K, Matsumoto H, Hara Y, Kutsukata N, Yamamoto Y (2007) Relationship between effect of polymyxin B-immobilized fiber and high-mobility group box-1 protein in septic shock patients. ASAIO J 53(3):324-328. https://doi. org/10.1097/MAT.0b013e3180340301

47. Ueno $T$, Ikeda $T$, Ikeda $K$, Taniuchi $H$, Suda $S$, Yeung MY, Matsuno N (2011) HMGB-1 as a useful prognostic biomarker in sepsis-induced organ failure in patients undergoing PMX-DHP. J Surg Res 171(1):183-190. https://doi.org/10.1016/j. jss.2009.11.708

48. Abe S, Hayashi H, Seo Y, Matsuda K, Kamio K, Saito Y, Usuki J, Azuma A, Kudo S, Gemma A (2011) Reduction in serum high mobility group box-1 level by polymyxin B-immobilized fiber column in patients with idiopathic pulmonary fibrosis with acute exacerbation. Blood Purif 32(4):310-316. https://doi. org/10.1159/000330325

49. Nemoto H, Nakamoto H, Okada H, Sugahara S, Moriwaki K, Arai M, Kanno Y, Suzuki H (2001) Newly developed immobilized polymyxin B fibers improve the survival of patients with sepsis. Blood Purif 19(4):361-368. discussion 368-369. https://doi. org/10.1159/000046966

50. Antonelli M, Ronco C (2015) Polymyxin B hemoperfusion in sepsis: growing body of evidence and occasional conflicting results. Blood Purif 39(1-3):I-II. https://doi.org/10.1159/000431018

51. Antonelli M, Fumagalli R, Cruz DN, Brienza N, Giunta F, EUPHAS Study Group (2010) PMX endotoxin removal in the clinical practice: results from the EUPHAS trial. Contrib Nephrol 167:83-90. https:// doi.org/10.1159/000315922

52. Cruz DN, de Cal M, Piccinni P, Ronco C (2010) Polymyxin-B hemoperfusion and endotoxin removal: lessons from a review of the literature. Contrib Nephrol 167:77-82. https://doi.org/10.1159/000315921

53. Early Use of Polymyxin BHitASCG (2014) Polymyxin B hemoperfusion in clinical practice: the picture from an unbound collaborative registry. Blood Purif 37(Suppl 1):22-25. https://doi. org/10.1159/000356835

54. Mitaka C, Tsuchida N, Kawada K, Nakajima Y, Imai T, Sasaki S (2009) A longer duration of polymyxin 
B-immobilized fiber column hemoperfusion improves pulmonary oxygenation in patients with septic shock. Shock 32(5):478-483. https://doi.org/10.1097/ SHK.0b013e3181a2a978

55. Ueno T, Sugino M, Nemoto $H$, Shoji $H$, Kakita A, Watanabe M (2005) Effect over time of endotoxin adsorption therapy in sepsis. Ther Apher Dial 9(2):128-136. https://doi. org/10.1111/j.1774-9987.2005.00230.x

56. Yamashita C, Hara Y, Kuriyama N, Nakamura T, Nishida O (2015) Clinical effects of a longer duration of polymyxin B-immobilized fiber column direct hemoperfusion therapy for severe sepsis and septic shock. Ther Apher Dial 19(4):316-323. https://doi. org/10.1111/1744-9987.12339

57. Suzuki H, Nemoto H, Nakamoto H, Okada H, Sugahara S, Kanno Y, Moriwaki K (2002) Continuous hemodiafiltration with polymyxin-B immobilized fiber is effective in patients with sepsis syndrome and acute renal failure. Ther Apher 6(3):234-240

58. Sakamoto Y, Mashiko K, Obata T, Matsumoto H, Hara Y, Kutsukata N, Yamamoto Y (2008) Effectiveness of continuous hemodiafiltration using a polymethylmethacrylate membrane hemofilter after polymyxin B-immobilized fiber column therapy of septic shock. ASAIO J 54(1):129-132. https://doi.org/10.1097/MAT.0b013e31815d2f01

59. Naka T, Shinozaki M, Akizawa T, Shima Y, Takaesu H, Nasu H (2006) The effect of continuous venovenous hemofiltration or direct hemoperfusion with polymyxin B-immobilized fiber on neutrophil respiratory oxidative burst in patients with sepsis and septic shock. Ther Apher Dial 10(1):7-11. https://doi. org/10.1111/j.1744-9987.2006.00339.x

60. Ebihara I, Hirayama K, Nagai M, Kakita T, Sakai K, Tajima R, Sato C, Kurosawa H, Togashi A, Okada A, Usui J, Yamagata K, Kobayashi M (2011) Angiopoietin balance in septic shock patients with acute lung injury: effect of direct hemoperfusion with polymyxin
B-immobilized fiber. Ther Apher Dial 15(4):349-354. https://doi.org/10.1111/j.1744-9987.2011.00963.x

61. Tsushima K, Kubo K, Koizumi T, Yamamoto H, Fujimoto K, Hora K, Kan-Nou Y (2002) Direct hemoperfusion using a polymyxin B immobilized column improves acute respiratory distress syndrome. J Clin Apher 17(2):97-102. https://doi.org/10.1002/ jca. 10019

62. Seo Y, Abe S, Kurahara M, Okada D, Saito Y, Usuki J, Azuma A, Koizumi K, Kudoh S (2006) Beneficial effect of polymyxin B-immobilized fiber column (PMX) hemoperfusion treatment on acute exacerbation of idiopathic pulmonary fibrosis. Intern Med 45(18):1033-1038

63. Abe S, Azuma A, Mukae H, Ogura T, Taniguchi H, Bando M, Sugiyama Y (2012) Polymyxin B-immobilized fiber column (PMX) treatment for idiopathic pulmonary fibrosis with acute exacerbation: a multicenter retrospective analysis. Intern Med 51(12):1487-1491

64. Yokoyama T, Tateishi K, Tsushima K, Agatsuma T, Yamamoto H, Koizumi T, Kubo K (2010) A case of severe ARDS caused by novel swine-origin influenza $(\mathrm{A} / \mathrm{H} 1 \mathrm{~N} 1 \mathrm{pdm})$ virus: a successful treatment with direct hemoperfusion with polymyxin B-immobilized fiber. J Clin Apher 25(6):350-353. https://doi. org/10.1002/jca.20252

65. Yatera K, Yamasaki K, Kawanami T, Tokuyama S, Ogoshi T, Kouzaki M, Nagata S, Nishida C, Yoshii C, Mukae H (2011) A case of successful treatment with polymyxin B-immobilized fiber column direct hemoperfusion in acute respiratory distress syndrome after influenza A infection. Intern Med 50(6):601-605

66. Kudo K, Binh NG, Manabe T, Co DX, Tuan ND, Izumi S, Takasaki J, Minh DH, Thuy PT, Van VT, Hanh TT, Chau NQ (2012) Clinical preparedness for severe pneumonia with highly pathogenic avian influenza A (H5N1): experiences with cases in Vietnam. Respir Investig 50(4):140-150. https://doi. org/10.1016/j.resinv.2012.08.005 